\title{
Multiobjective Optimization of Large-Scale EVs Charging Path Planning and Charging Pricing Strategy for Charging Station
}

\author{
Weicheng Hou $\mathbb{D}^{1,2}$ Qingsong Luo $\mathbb{D}^{2,3}$ Xiangdong $W u\left(\mathbb{D},{ }^{2,3}\right.$ Yimin Zhou $\mathbb{D}^{2}$, \\ and Gangquan Si iD \\ ${ }^{1}$ Xi'an Jiaotong University, Xi'an 710049, China \\ ${ }^{2}$ Shenzhen Institutes of Advanced Technology, Chinese Academy of Sciences, Shenzhen 518055, China \\ ${ }^{3}$ University of Chinese Academy of Sciences, Beijing 100049, China \\ Correspondence should be addressed to Yimin Zhou; ym.zhou@siat.ac.cn
}

Received 21 August 2020; Revised 31 January 2021; Accepted 14 February 2021; Published 25 February 2021

Academic Editor: Ramon Costa-Castelló

Copyright $(2021$ Weicheng Hou et al. This is an open access article distributed under the Creative Commons Attribution License, which permits unrestricted use, distribution, and reproduction in any medium, provided the original work is properly cited.

With the increasing number of electric vehicles (EVs), the charging demand of EVs has brought many new research hotspots, i.e., charging path planning and charging pricing strategy of the charging stations. In this paper, an integrated framework is proposed for multiobjective EV path planning with varied charging pricing strategies, considering the driving distance, total time consumption, energy consumption, charging fee such factors, while the charging pricing strategy is designed based on the objectives of maximizing the total revenues of the charging stations and balancing the profits of the charging stations. First, the energy consumption model of EVs, the M/M/S queuing model of charging stations, and the charging model of charging piles are established. A novel charging path planning algorithm is proposed based on bidirectional Martins' algorithm, which can assist EV users to select charging stations and plan charging paths. Then, a particle swarm optimization (PSO) algorithm is applied to solve the optimal solution of charging station pricing designation. Finally, the method proposed in the paper is simulated on the street map of Shenzhen to verify the efficacy of the multiobjective charging path planning for EVs and the feasibility of the charging pricing strategy.

\section{Introduction}

EVs can reduce the emissions of harmful substances generated by the use of traditional fossil energy sources, so it is one of the promising alternatives to deal with the future energy crisis and environmental pollution [1]. However, the EVs have the problem of longer battery charging time and shorter driving range than petrol vehicles, which could result in "charging anxiety" and "range anxiety" for the EV users $[2,3]$. Therefore, how to use EVs wisely is a concern of EV users and researchers. Considering factors such as driving distance, driving duration, energy consumption, and charging fee, EV users would select a reasonable charging path to alleviate their "range anxiety" [4].

EV charging path planning is a multiobjective problem. To ensure the shortest driving duration to the charging station and the least charging cost under the premise that the battery is not depleted, the charging decision of EV users can be modeled as an energy-aware shortest path problem (ESPP) on a traffic network diagram with a virtual arc [5]. Considering the total driving time and the total energy consumption of the $\mathrm{EV}$, the problem is modeled as a bilevel formulation of the discrete multiclass equilibrium network design, where the upper level is solved using a GA and the lower level is a multiclass user equilibrium (UE) traffic assignment model [6]. The elevation factor is considered to calculate the energy consumption [7]. The charging time of each candidate charging station and the total travelling time to the destination are considered in the charging path planning [8]. Deep reinforcement learning (DRL) can be applied in the charging scheduling for EVs with the optimization objective to minimize the total cost of EV users, including charging time and 
charging fares, under the condition of charging availability and fluctuated electricity prices [9].

The multiobjective decision-making problem has been studied extensively [10]. A practical ISE-IITOPSIS method is proposed to solve the multifeature identification problem [11]. The most direct and simple method of solving the multiobjective path is the weighted method, which aggregates multiple objectives into a single objective problem through assigning weight to each objective [12]. However, this method requires normalization of the parameters and fine-tuning weights. Classic algorithms, i.e., graph-based theory, can also be applied. For example, Yen's algorithm is used to ensure that travelling time and delay time caused by traffic congestion are minimized [13]. Besides, the heuristic algorithms (i.e., the multiobjective intelligent evolutionary algorithm) are applied to solve the Pareto solution set of the shortest path under constraints. For example, a hybrid variable domainsearch algorithm is proposed to solve the extended $\mathrm{EV}$ routing problem, via the EV battery swap station location routing with stochastic demands [14]. The classic algorithm can obtain an accurate Pareto solution. However, with the consideration of a city-level traffic network based on the huge amount of nodes and edges, heuristic algorithms are feasible to solve the optimal solution [15]. Martins' algorithm [16] is a classic multiobjective shortest path algorithm based on the Dijkstra algorithm, but it has to search the entire network to get the optimal Pareto solution set. Therefore, an improved method based on Martins' algorithm is proposed [17], including setting interruption conditions and bidirectional search (i.e., search from the end point and start point simultaneously). Simulation experiments demonstrate that the computational efficiency can be significantly improved in a large-scale transportation network.

The pricing mechanism of EV charging stations should also be investigated. The commonly studied pricing strategies mainly include fixed electricity price [18], time-of-use (TOU) price [19], and real-time price (RTP) [20]. RTP is a method of dynamically adjusting electricity price via the relationship between the power system supply and demand [21]. In the power system, a fundamental model is proposed for continuous-time scheduling and marginal pricing of energy generation storage in day-ahead power systems' operation [22]. In the home energy management system (HEMS), a new charging and discharging strategy for EVs with energy price control is proposed for the best benefits of EV owners. RTP can also be used in the pricing strategy of charging stations $[23,24]$.

A multiobjective comprehensive stand-alone solution is proposed [25] to use TOU to intelligently regulate the charging/discharging activities of EVs, with the purpose of cutting peaks and filling valleys of loads and operating costs of power system reduction. Further, a charging pricing strategy based on regional division and time division is proposed, where the charging prices between different areas of the city are optimized to guide EV users to select to charge in the area with more charging stations. Then, from the perspective of consumer satisfaction, grid company profits, and grid load variation, a TOU optimization model for EVs is constructed [26]. On the contrary, a pricing scheme is proposed assigning a per-session price to each charging session to accommodate the energy cost, demand charge, and system congestion [27]. The current pricing strategies for the charging stations mainly focus on the fixed electricity price or TOU price, which may cause congestion in local charging stations and undesirable peak loads on the grid. The RTP can guide the charging behavior of EVs to avoid invalid charging infrastructure investment and to maximize the revenue of charging stations [28,29].

Studies demonstrate that the charging price can affect the results of EV charging path planning and the selection of charging stations. The charging path planning can be considered as a multiobjective problem with driving distance, charging time, energy consumption, and charging fee. In this paper, these four objectives are combined together to plan the charging path for EVs based on bidirectional Martins' algorithm. The driving time, charging time, and queuing time of EVs are used to estimate the total time consumption, while energy consumption of EVs and the charging price of the charging station are used to estimate the charging fee. The charging decision-making of EV users is stimulated through large-scale EV charging path planning. Besides, the PSO algorithm along with the charging path planning is used to solve the optimal charging price of the charging station so that the profit of the charging station is maximized, while the standard deviation of the profit of each charging station is minimized. The proposed method includes precalculation, query, and selection of three stages. In the precalculation stage, the topology of the road network and the locations of the charging stations are static and known. In the query stage, the heuristic items are added to each objective, using precomputed data to estimate the cost to the charging station. The main contributions of the paper are summarized as follows:

(1) An improved multiobjective charging path planning algorithm based on bidirectional Martins' algorithm is proposed to solve the $\mathrm{EV}$ charging path problem. The bidirectional Martins' algorithm is improved with adding heuristic items to the dimensions of distance, time, and energy consumption to estimate the cost to the end point, as well as the pruning method to speed up the calculation.

(2) A charging selection-based adaptive pricing strategy is established to maximize the total revenues of the charging stations, while balancing the profit of each charging station. The results of large-scale EV charging path planning is used to simulate the selection of charging stations with different prices for EV users, and the PSO algorithm is applied to solve the optimal solution of the charging station charging price.

(3) A series of simulation experiments have been performed with the Shenzhen city street map, including different roads, to verify the effectiveness of the model.

The remainder of the paper is organized as follows. In Section 2, the energy consumption model, the charging 
model, and the queuing model are presented; afterwards, an integer nonlinear programming model of charging path planning is developed. In Section 3, a multiobjective charging path planning algorithm based on bidirectional Martins' algorithm is proposed with heuristic and pruning techniques and driver's preference to solve the path planning problem and recommend the charging path to EV users. In Section 4, a charging selection-based pricing strategy for charging stations is proposed to maximize their revenues and balance the revenue of each charging station. In Section 5 , simulation experiments are performed to illustrate the application and effectiveness of the proposed method. Conclusion and future works are given in Section 6.

\section{Problem Formulation}

In this section, the four optimization objectives, i.e., driving distance, total time consumption (summation of driving time, waiting time, and charging time), energy consumption, and charging fee are formulated as a mathematical model.

Certain assumptions are made in this paper. (1) EVs are fully charged when they are charged at the charging stations. (2) Energy consumption is a positive value, and the recuperation when braking or driving down a slope is neglected.

Considering a directed road network $G(N, A), N$ is the set of nodes, and $A$ is the set of links, and the link from node $i$ to node $j$ is defined as $(i, j) \in A$. The charging stations in a graph are defined as $\mathbf{C S}=\left\{c_{1}, c_{2}, \ldots, c_{k}, \ldots c_{K}\right\}$ and $\mathbf{C S} \subset N$ where each charging station is denoted as node $c_{k}$, and $K$ is the number of the charging stations. The total time consumption of a charging path $\zeta$ is $t(\varsigma)=t_{d}(\varsigma)+t_{w}\left(c_{k}\right)+t_{c}\left(c_{k}\right)$, where $t_{d}(\varsigma)$ represents the driving time, $t_{w}\left(c_{k}\right)$ represents the waiting time at the specific charging station $c_{k}$, and $t_{c}\left(c_{k}\right)$ represents the charging time at the $c_{k}$ charging station. Table 1 lists the related parameters in the models.

\section{Energy Model of the EV}

According to [30], the energy consumption of an EV is mainly related to the travelling distance $d_{i j}$ and the average speed $v_{i j}=d_{i j} / t_{d}(i, j)$. The energy consumption $e_{i j}\left(v_{i j}, d_{i j}\right)$ of the link $(i, j)$ under the condition of average speed $v_{i j}$ and distance $d_{i j}$ can be written as

$$
e_{i j}\left(v_{i j}, d_{i j}\right)=\frac{d_{i j}}{\eta_{\text {motor }}}\left(0.5 \rho C w v_{i j}^{2}+\mu m g \cos \theta+m g \sin \theta\right)+d_{i j} \frac{P_{\text {add }}}{v_{i j}},
$$

where the parameters $\rho, C, w, \mu, g$, and $P_{\text {add }}$ represent the density of the air, drag coefficient, cross-sectional area, rolling resistance coefficient, gravity acceleration, and average additional energy consumption, respectively. The energy consumption function $e_{i j}$ is a convex function, while Figure 1 depicts the energy consumption curve with the parameters $\quad d_{i j}=1 \mathrm{~km}, \quad \eta_{\text {motor }}=0.9, \quad \rho=1.29 \mathrm{~kg} / \mathrm{m}^{3}$, $C=0.35, w=2.3 \mathrm{~m}^{2}, \mu=0.015, m=1600 \mathrm{~kg}, g=9.81 \mathrm{~m} / \mathrm{s}^{2}$, and $P_{\text {add }}=1.5 \mathrm{~kW}$.

It is noted that the developed energy consumption model is simplified by neglecting certain factors such as energy recovery, while braking. However, this does not affect the effectiveness of the method discussed later in this paper.

3.1. Charging Model. The charging time is calculated by the charging power of the charging station and the current SOC of EVs when EVs arrive at the charging stations. Most studies assume that the charging output power of the charging stations is the same among the charging stations. In practice, the charging power provided by the stations is varied, which could affect the EV charging time.

Assuming that the charging pile power of the charging station $c_{k}$ is $P_{c}\left(c_{k}\right)$, the charging time $t_{c}$ of fully charged is calculated as

$$
\begin{aligned}
t_{c}\left(c_{k}\right) & =\frac{E_{0}-E_{\text {rem }}}{P_{c}\left(c_{k}\right) \cdot \eta_{c_{k}}}, \\
E_{\text {rem }} & =\operatorname{SOC} \cdot E_{0}-E_{\text {road }}, \\
E_{\text {road }} & =\sum_{(i, j) \in A} e_{i j} \cdot x_{i j}, \\
x_{i j} & = \begin{cases}1, & \text { if link }(i, j) \text { is on the path, } \\
0, & \text { else, }\end{cases}
\end{aligned}
$$

where $E_{\text {road }}$ is the required energy for the EV travelling to the charging station. The charging pile power $P_{c}\left(c_{k}\right)$ varies depending on the charging level of the charging station. Four levels of the charging stations can be defined based on the facilities in the current market, see in Table 2.

3.2. Queuing Model. The state of the EV charging station can be represented with the Markov chain by incorporating the state information, as shown in Figure 2 [31]. The arrival of the $\mathrm{EV}$ is considered as a Poisson process with an arrival rate of $\lambda$, and each charging pile is considered to serve with an exponential service rate $\mu$. A multiserver queue with $s$ identical servers is considered here.

According to the queuing theory, the balance of each state in the queue model can be solved. In each state, the leaving rate based on arrival is equal to the entering rate based on departure, i.e.,

$$
\begin{aligned}
\lambda \pi_{0} & =\mu \pi_{1}, \\
(\lambda+\mu) \pi_{1} & =\lambda \pi_{0}+2 \mu \pi_{2}, \\
(\lambda+2 \mu) \pi_{2} & =\lambda \pi_{1}+3 \mu \pi_{3}, \\
\cdots & \\
(\lambda+(k-1) \mu) \pi_{k-1} & =\lambda \pi_{k-2}+k \mu \pi_{k},
\end{aligned}
$$

where $\pi_{k}$ denotes the probability that the number of charged EVs in the charging station is $k$. The left side of equation (3) is the leaving rate, and the entering rate is described on the right side. Based on the state transition diagram given in equation (3), the corresponding transition matrix is written as 
TABLE 1: The involved parameters in the EV charging path planning.

\begin{tabular}{|c|c|}
\hline Variables & Specification \\
\hline$N$ & Set of nodes of the road network \\
\hline$K$ & Number of the charging stations \\
\hline$d(\varsigma)$ & EV driving distance of the planned path $\varsigma$ \\
\hline$d_{i j}$ & Driving distance from node $i$ to node $j$ of the EV \\
\hline$t_{c}\left(c_{k}\right)$ & Charging time at the charging station $c_{k}$ \\
\hline$p_{c_{k}}$ & Charging price of the charging station $c_{k}$ \\
\hline$E_{0} c_{k}$ & The battery capacity of the EV \\
\hline$x_{i j}$ & A bool integer denoting whether the link $(i, j)$ is on the planned charging path \\
\hline$L(i)$ & The node $i$ label denoting the cost from the origin node to node $i$ and the previous node label \\
\hline$A$ & Set of links of the road network \\
\hline$c_{k}$ & The $k^{\text {th }}$ charging station \\
\hline$\hat{t}(\varsigma)$ & Total time consumption of the planned path $\varsigma$ \\
\hline$v_{i j}$ & Average velocity of the link $(i, j)$ \\
\hline$t_{w}\left(c_{k}\right)$ & Waiting time at the charging station $c_{k}$ \\
\hline fee $(\varsigma)$ & Charging fee of the planned path $\varsigma$ for the EV \\
\hline$E_{\text {rem }}$ & The remaining battery energy when the EV arrives at the charging station \\
\hline$r\left(c_{k}\right)$ & Charging revenue of the $c_{k}$ charging station \\
\hline$P$ & The driver's preference to select a charging path from a Pareto set of paths \\
\hline CS & Set of all charging stations \\
\hline$\varsigma$ & A planned charging path for the EV \\
\hline$e(\varsigma)$ & Energy consumption of the planned path for the EV \\
\hline$t_{d}(i, j)$ & Driving time from node $i$ to node $j$ of the EV \\
\hline$e_{i j}$ & Energy consumption from node $i$ to node $j$ of the EV \\
\hline SOC & Initial SOC when EV makes a query for charging path planning \\
\hline$\eta_{c_{k}}$ & The charging efficiency of the charging piles at the $c_{k}$ charging station \\
\hline$C(i, j)$ & Cost vector from the node $i$ to the node $j$ when planning the path \\
\hline
\end{tabular}

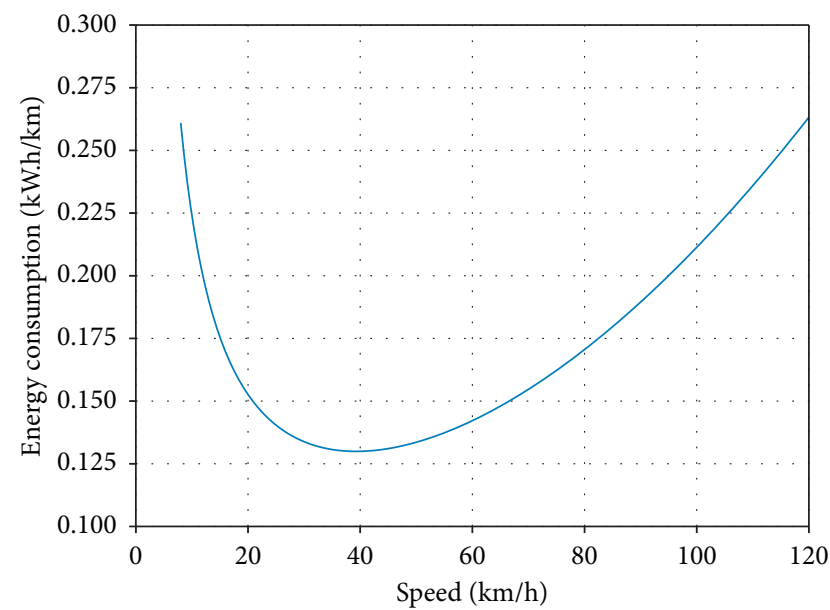

FIGURE 1: Energy consumption function of the EV.

TABLE 2: The current charging levels of the charging stations in the market.

\begin{tabular}{lc}
\hline Charging level & Charging power \\
\hline Standard & $2-5 \mathrm{~kW}$ \\
Midaccelerated & $5-16 \mathrm{~kW}$ \\
Accelerated & $16-30 \mathrm{~kW}$ \\
Fast & $30-350 \mathrm{~kW}$ \\
\hline
\end{tabular}
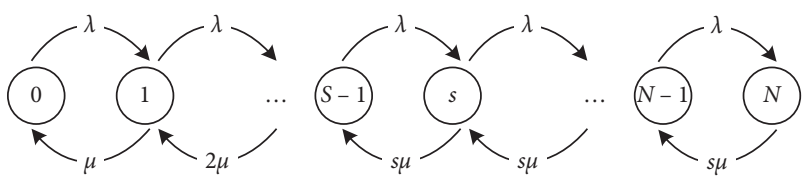

FIGURE 2: Markov chain of the EV charging station.

$$
M=\left[\begin{array}{cccccccc}
-\lambda & \lambda & 0 & 0 & & & & \\
\mu & -(\lambda+\mu) & \lambda & 0 & 0 & & & \\
0 & 2 \mu & -(\lambda+2 \mu) & \lambda & 0 & 0 & & \\
0 & 0 & 3 \mu & -(\lambda+3 \mu) & \lambda & 0 & 0 & \\
\cdots & \cdots & \cdots & \cdots & \cdots & \ldots & \ldots & \ldots \\
& & & & & s \mu & -(\lambda+s \mu) & \lambda
\end{array}\right] .
$$

The stationary condition related to the Markov chain model is given by

$$
\bar{\pi} * M=\overline{0},
$$

where $\bar{\pi}=\left[\pi_{0}, \pi_{1}, \ldots, \pi_{N}\right]$ is the queue length distribution under the condition of the stationary state, and the subscript ' $N$ is the state number. The general recursive form of the stationary distribution (state $k$ ) is derived as 


$$
\pi_{k}= \begin{cases}\frac{1}{k !}\left(\frac{\lambda}{\mu}\right)^{k} \pi_{0}, & \text { if } k \leq s, \\ \left(\frac{1}{s}\right)^{k-s} \frac{1}{s !}\left(\frac{\lambda}{\mu}\right)^{k} \pi_{0}, & \text { if } k \geq s,\end{cases}
$$

where $s$ is the number of the charging piles. Therefore, the mean queue length is given by

$$
E(\text { len })=\sum_{k=s}^{N}(k-s) \pi_{k}
$$

and the mean waiting time in the queue is given by

$$
E\left(t_{w}\right)=\frac{E(\text { len })}{\lambda\left(1-\pi_{N}\right)} .
$$

3.3. Constraint Conditions. The flow balance constraints are applied here. For the origin node, the outgoing flows of all links are one more than the incoming flow. For the destination node, the incoming flows of all links are one more than the outgoing flow. For other nodes, the incoming flow is equal to the outgoing flow. So, the details of the flow balance constraint are expressed as follows:

$$
\sum_{(i, j) \in A} x_{i j}-\sum_{(j, i) \in A} x_{j i}=b_{i}, \quad \text { for each } i \in N,
$$

where $b_{i}$ denotes the flow balance of the node $i$, classified into three types, the starting node $i=1$, ending node $i=c_{k}$, and passing nodes $i \neq 1, c_{k}$ :

$$
\begin{aligned}
& b_{1}=1, \\
& b_{c}=-1, \\
& b_{i}=0, \quad \text { for } i \neq 1, c_{k},
\end{aligned}
$$

where $c_{k}$ denotes one of the charging stations, i.e., $c_{k} \in \mathbf{C S}$. Considering the limitation of the battery capacity in EVs, the remaining driving distance is limited, expressed as

$$
\sum_{(i, j) \in A} e_{i j} \cdot x_{i j}<\mathrm{SOC} \cdot E_{0} .
$$

3.4. Optimization Objectives. Given a charging path $\varsigma$, with the target charging station $c_{k}$, the queuing time, charging time, and charging fee can thus be estimated for this path. The total driving distance $d(\varsigma)$, time consumption $t(\varsigma)$, total energy consumption $e(\varsigma)$, and charging fee fee $(\varsigma)$ of the planned charging path $\varsigma$ with the target charging station $c_{k}$ are written as

$$
\begin{aligned}
d(\varsigma) & =\sum_{(i, j) \in A} d_{i j} \cdot x_{i j}, \\
t(\varsigma) & =t_{c}\left(c_{k}\right)+t_{w}\left(c_{k}\right)+\sum_{(i, j) \in A} t_{d}(i, j) \cdot x_{i j}, \\
e(\varsigma) & =\sum_{(i, j) \in A} e_{i j} \cdot x_{i j}, \\
\text { fee }(\varsigma) & =p_{c}\left(c_{k}\right) \cdot \frac{E_{0}-E_{\mathrm{rem}}}{\eta_{c_{i}}} .
\end{aligned}
$$

Note that $d_{i j}>0, t_{d}(i, j)=\left(d_{i j} / v_{i j}\right)>0$, and $e_{i j}>0$. The optimized objective of the model is formulated as

$$
\min \{d(\varsigma), e(\varsigma), t(\varsigma), \text { fee }(\varsigma)\}
$$

The above multiobjective optimization is an integer nonlinear programming problem with the decision variables of $c_{k}$ and $x_{i j}$ of each link $(i, j)$, which has a high temporal complexity. How to select the optimal paths is already an intrigue problem.

\section{Solution Algorithm}

In this section, the basic Martins' algorithm (MA) [16] and the bidirectional Martins' algorithm (BMA) [17] are introduced first, and an improved EV heuristic bidirectional Martins' algorithm (EHBMA) is designed to optimize the objectives described in equation (13).

4.1. The Basic Algorithm. In order to develop the algorithm, certain definitions are introduced first, where the cost vector of the link $(i, j)$ is denoted as $C(i, j)=\left(d_{i j}, t_{d}(i, j), e_{i j}\right)$, the node $i$ label is denoted as $L(i)=[i, C(o, i)$, previous_label], and $o$ is the origin point of the path.

Definition 1. Given two cost vectors of link $C(i, j)$ and $C(m, n), C(i, j)$ dominates $C(m, n)$ if and only if $d_{i j} \leq d_{m n}$, $t_{d}(i, j) \leq t_{d}(m, n)$, and $e_{i j} \leq e_{i j}$ and at least one inequality is strict.

Definition 2. Given two paths $\varsigma_{i}$ and $\varsigma_{j}$ containing a series of nodes $\varsigma_{i}=\left(o, i, \ldots, m, c_{k}\right)$ and $\varsigma_{j}=\left(o, j, \ldots, n, c_{l}\right)$, with two target charging station $c_{k}$ and $c_{l}$, $\varsigma_{i}$ dominates $\varsigma_{j}$ if and only if $d_{\varsigma_{i}} \leq d_{\varsigma_{j}}, t\left(\varsigma_{i}\right) \leq t\left(\varsigma_{j}\right), e\left(\varsigma_{i}\right) \leq e\left(\varsigma_{j}\right)$, and fee $\left(\varsigma_{i}\right) \leq$ fee $\left(\varsigma_{j}\right)$ and at least one inequality is strict. A path $\varsigma$ is a nondominated path if and only if $\zeta$ is an optimal path in at least one objective, and it is not dominated by any other paths.

Definition 3. Given two node labels $L(i)$ and $L(j)$ of node $i$ and node $j, L(i)$ dominates $L(j)$ if and only if $i=j$ and $C_{o i}$ dominates $C_{o j}$.

In the original MA, new label $L_{\text {new }}(i)$ is added to the node $i$ if and only if the label $L_{\text {new }}(i)$ is not dominated by other labels of the node $i$. Further, the bidirectional MA (BMA) can limit the search area by formulating a termination condition and searching the network bidirectionally. The pseudocodes of the MA and BMA are shown in Algorithms 1 and 2, respectively, where the target point is represented by $t$, and $\left[\min _{i}(T)\right]$ represents the minimum value of each objective value of all labels in $T$, forming the vector $\left[\min _{1}(T), \min _{2}(T), \ldots, \min _{\text {objective_num }}(T)\right]$.

\subsection{Improved EV Heuristic Bidirectional Martins' Algorithm} (EHBMA). Based on the BMA, considering the EV charging path planning scenario, the EHBMA is proposed in this paper, including three stages. In the $1^{\text {st }}$ stage, the map data is preprocessed, and the shortest distance from each charging station to each node is calculated and stored. In the $2^{\text {nd }}$ stage, considering that EV users hardly travel too far to charge in 
Create a list $T$

The initial element in the list $T$ is the label of the starting point $o,[o,(0,0,0)$, NULL $]$

Create the path set PATH_RESULT

(1) While $T$ is not empty

(2) Get the minimum node label in $T, L$ (node)

(3) For the adjacent node, new_node

(4) Calculate the label of the adjacent point $L$ (new_node)

(5) If $L$ (new_node) is not dominated by other node labels of new_node, and not dominated by labels in PATH_RESULT

(6) Then put $L$ (new_node) into list $T$

(7) If new_node $=t$

(8) Then get the previous nodes of new_node, and put the path nodes into PATH_RESULT

Algorithm 1: Basic Martins' algorithm.

Create a forward list $T_{-}$FOR and a backward list $T_{-}$BACK

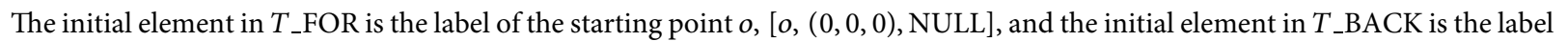
of the end point $t,[t,(0,0,0)$, NULL $]$

Create the path set PATH_RESULT

The initial search direction is forward direction $=0$

(1) While $\left[\min _{i}\left(T_{-}\right.\right.$FOR $\left.)\right]+\left[\min _{i}\left(T_{-}\right.\right.$BACK $\left.)\right]$is not dominated by PATH_RESULT

(2) If direction $=0$, Then get the minimum node label $L$ (node) in $T_{\text {_FOR }}$

(3) For the child adjacent node of node, new_node

(4) Calculate the node label of the adjacent point, $L_{-}$FOR (new_node)

(5) If $L_{-}$FOR (new_node) is not dominated by other node labels of new_node, Then put $L_{-}$FOR(new_node) into $T \_F O R$

(6) If direction $=1$, Then get the minimum node label $L$ (node) in $T \_$BACK

(7) For the parent adjacent node of node, new_node

(8) Calculate the node label of the adjacent point, L_BACK (new_node)

(9) If $L_{-} B A C K$ (new_node) is not dominated by other node labels of new_node, Then put $L_{-} B A C K$ (new_node) into T_BACK

(10) If the label of new_node is in the list of reverse direction, Then obtain the final path by return the previous node label in L_FOR and $L_{-}$BACK, and add the path intoPATH_RESULT

(11) Change the direction to the opposite

Algorithm 2: Bidirectional Martins' algorithm.

general situations, the three closest charging stations are preselected as alternatives according to the preprocessed map data, then the multiobjective path planning process is performed among the selected charging stations to achieve candidate paths. In the $3^{\text {rd }}$ stage, the final charging path is selected according to EV user's preference. Moreover, in order to speed up the path planning process, the heuristic method and pruning method are introduced in the EHBMA.

4.2.1. Heuristic Method. To estimate the remaining distance, travelling time, and required energy consumption to the charging station, the heuristic items of $f_{d}(i), f_{t}(i)$, and $f_{e}(i)$ are added to the original label of node $i$. After adding the heuristic items, the nodes with calculated closer distance to the target charging station, shorter travelling time, and less energy consumption are visited first so that EVs can reach the target charging station faster with fewer nodes traversed than the original algorithm.

The heuristic items of the forward search and backward search are different, assuming that the direction $=0$ represents the forward search (i.e., from origin node $o$ to the target charging station $c_{k}$ ), and direction $=1$ represents the opposite direction. In the distance dimension,

$$
f_{d}(i)=\left\{\begin{array}{l}
\operatorname{SPL}\left(i, c_{k}\right), \quad \text { direction }=0, \\
\operatorname{EuDist}(o, i), \quad \text { direction }=1,
\end{array}\right.
$$

where $\operatorname{SPL}\left(i, c_{k}\right)$ is the shortest distance from the current node $i$ to the charging station $c_{k}$ and $\operatorname{EuDist}(o, i)$ is the Euler distance from the origin node $o$ to the current node $i$. In the time dimension, it has

$$
f_{e}(i)= \begin{cases}\frac{\operatorname{SPL}\left(i, c_{k}\right)}{\bar{v}}, & \text { direction }=0, \\ \frac{\operatorname{EuDist}(o, i)}{\bar{v}}, & \text { direction }=1,\end{cases}
$$

where $\bar{v}$ denotes the past average driving speed of the EV. In the energy consumption dimension,

$$
f_{e}(i)= \begin{cases}\operatorname{SPL}\left(i, c_{k}\right) \cdot \bar{e}, & \text { direction }=0, \\ \operatorname{EuDist}(o, i) \cdot \bar{e}, & \text { direction }=1,\end{cases}
$$

where $\bar{e}$ denotes the past average energy consumption of the $\mathrm{EV}$. Therefore, the new node label of node $i$ with heuristic items can be calculated as 


$$
\begin{aligned}
& L_{-} \operatorname{HEU}(i)=\left\{\begin{array}{lr}
{[i, C(o, i), C(o, i)+H(i), \text { previous_label }],} & \text { direction }=0, \\
{\left[i, C\left(i, c_{k}\right), C\left(i, c_{k}\right)+H(i), \text { previous_label }\right],} & \text { direction }=1,
\end{array}\right. \\
& H(i)=\left(f_{d}(i), f_{t}(i), f_{e}(i)\right) \text {. }
\end{aligned}
$$

Definition 4. $L_{-} \operatorname{HEU}(i)$ dominates $L_{-} \operatorname{HEU}(j)$ if and only if the two conditions hold, 1) $i=j$ and 2) $C(o, i)+H(i)$ dominates $C(o, j)+H(j)$ if direction $=0$ or $C\left(i, c_{k}\right)+H(i)$ dominates $C\left(j, c_{k}\right)+H(j)$ if direction $=1$.

4.2.2. Pruning Method. If the label $L_{-} \operatorname{HEU}(i)$ of node $i$ is dominated by the labels of the existing path from the origin node to the target node or the estimated energy consumption $f_{e}(i)$ of the node $i$ is greater than the remaining power of the EV, the node $i$ will not be traversed in the algorithm. By pruning the traversed node, algorithm efficiency can be further improved.

4.2.3. The Driver's Preference. A set of Pareto optimal solutions can be achieved after performing the proposed algorithm, and the most optimal path can be selected according to the driver's behavior preferences, described as

$$
P=\frac{(\alpha \cdot d(\varsigma)+\beta \cdot t(\varsigma)+\gamma \cdot e(\varsigma)) \cdot(\mu \cdot \mathrm{fee}(\varsigma))}{\lambda_{c_{k}}},
$$

where $\alpha, \beta, \gamma$, and $\mu$ denote the preference factors. By setting appropriate preference factors, the driver can be recommended the most suitable charging path. $\lambda_{c_{k}}$ is a comprehensive metric of preference for the path to the charging station $c_{k}$, which depends on the factors of the number of recreation areas and living facilities around the charging station $c_{k}$. The higher the value, the more the inclination of the driver to select this charging station $c_{k}$.

In summary, the multiobjective charging path planning algorithm EHBMA is described with Algorithm 3.

\section{Charging Selection-Based Adaptive Pricing Strategy (CSBAP)}

In this section, the CSBAP is proposed to determine the charging price of the charging stations, so as to maximize the overall profits and balance the profits of each charging station. The details are explained as follows.

For charging station $c_{k}$, the revenue $r\left(c_{k}\right)$ can be calculated as

$$
r\left(c_{k}\right)=\sum_{\mathrm{EV}} p_{c}\left(c_{k}\right) \cdot \frac{E_{0}-E_{\mathrm{rem}}}{\eta_{c_{k}}} .
$$

The profits of the charging stations may be influenced by its location which could result in unbalanced profits' distribution and unable to maximize the total profits, while appropriate prices' setting can attract more EVs to balance the profits' distribution. From the analysis of the previous section, the charging selection of EV users can be influenced by the charging price. Therefore, the CSBAP is proposed accordingly along with the proposed charging path planning algorithm EHBMA and PSO algorithm, in which the EHBMA is used to simulate the selection of charging stations with different prices for EV users, and the PSO is applied to adjust the charging prices adaptively. The pseudocodes of the PSO algorithm are shown in Algorithm 4. Here, part ${ }_{i}$ denotes the $i^{\text {th }}$ particle, pos denotes the decision variable vector, and the velocity $V$ represents the changing rate of pos. The fitness function fit (pos) denotes the objective value to evaluate the equality of pos, pBest and gBest denote the best-known position of the single particle and the bestknown position of the whole swarm, respectively, rand represents a random number in $[0,1], \varepsilon$ denotes the minimum deviation of pos of the two iterations, and MAX denotes the maximum number of iterations.

With the combined PSO algorithm and the proposed EHBMA, the pricing strategy for single charging station and multiple charging stations is designed separately. First, the relationship between the charging price and the revenue of a single charging station is studied. Assuming that the charging prices of all charging stations are fixed, except for the objective charging station, the fitness function can be defined as fit $=r\left(c_{k}\right)$, where $r\left(c_{k}\right)$ denotes the revenue of the charging station $c_{k}$. The position of the particle can be defined as the charging price $p_{c}\left(c_{k}\right)$ of the charging station $c_{k}$ to acquire the optimal charging price gBest for the charging station.

The pricing strategy for multiple charging stations is further investigated. The fitness function is defined as the summation of the total revenues of all charging stations and the standard deviation of revenues fit $=\sum_{c_{i}}^{C} r\left(c_{k}\right)-\rho \cdot \operatorname{std}\left(\widehat{r}\left(c_{k}\right)\right)$, where $\operatorname{std}\left(\widehat{r}\left(c_{k}\right)\right)$ denotes the standard deviation of the revenue of all charging stations and $\rho$ denotes the coefficient to make the trade-off between two factors. The positions of the particles are defined as the charging price vector of all charging stations. The fitness function is designed to maximize the total profits of the charging stations, while balancing the profit of each charging station. The pricing system is adopted with the real-time charging path planning simulation.

\section{Simulation Results}

A road network dataset of Shenzhen city is used to verify the effectiveness of the model. The dataset is collected from the OpenStreetMap, which includes residential street, tertiary road, secondary road, primary road, trunk road, and motorway, while each road is composed of multiple location nodes with longitude and latitude of the WGS84 coordinate system. Moreover, the location of the charging stations, oneway or two-way characteristics, road length, and speed limitation are given in the dataset.

The road networks in Shenzhen is shown in Figure 3(a), and the speed limit of different road types are listed in 


\section{Initialization}

Initialize the average velocity $v_{i j}$ of each road link $(i, j)$ based on the traffic condition.

Calculate the energy consumption $e_{i j}$ and driving time $t_{d}(i, j)$ of the road link $(i, j)$ based on the energy consumption model and average velocity.

Calculate the shortest distance from each charging station to each node on the map and save the result as a dictionary preprocessing in which the keys are charging station nodes and the values are all other nodes on the map.

Initialize the location of the $\mathrm{EV}, o$.

Charging Path Planning

Select three charging stations cs_nearest closest to the current location $o$ by query the data preprocessing.

(1) For $c_{k} \in$ cs_nearest

(2) Create a forward list $T_{-}$FOR and a backward list $T_{-}$BACK

(3) The initial elements in $T_{-}$FOR and $T_{-}$BACK are the label of the starting point $o,[o,(0,0,0),(0,0,0)$, NULL], and the label of the end point $c_{k},\left[c_{k},(0,0,0),(0,0,0)\right.$, NULL $]$

(4) Create the path set PATH_RESULT

(5) The initial search direction is forward direction $=0$

(6) While $\left[\min _{i}\left(T_{-}\right.\right.$FOR $\left.)\right]+\left[\min _{i}\left(T_{-}\right.\right.$BACK) $)$is not dominated by the label in PATH_RESULT

(7) If direction $=0$, Then get the minimum node label $L_{-} \mathrm{HEU}$ (node) in $T_{-}$FOR

(8) For the child adjacent node of node, new_node

(9) Calculate the heuristic items $H$ (new_node) by query preprocessing

(10) Calculate the node label of the adjacent node, L_HEU_FOR (new_node)

(11) If $L_{-}$HEU_FOR (new_node) is not dominated by other node label of new_node, Then put $L_{-}$HEU_FOR (new_node) into T_FOR

(12) If direction $=1$, Then get the minimum node label $L_{-} H E U$ (node) in $T_{-}$BACK

(13) For the parent adjacent node of node, new_node

(14) Calculate the heuristic items $H$ (new_node)

(15) Calculate the node label of the adjacent node, $L_{-}$HEU_BACK (new_node)

(16) If $L_{-} H_{E U}$ BACK (new_node) is not dominated by other node label of new_node, Then put $L_{-} H_{E U} \_B A C K$ (new_node) into $T_{-} \mathrm{BACK}$

(17) If the label of new_node is in the list of reverse direction, Then get a path by return the previous node label in $L_{-} H E U_{-}$FOR and $L_{-} H_{E U}$ BACK, and add the path into PATH_RESULT

(18) Change direction to the opposite

\section{(19) Pareto Path Selection}

(20) For $\varsigma \in$ PATH_RESULT, its end point is a charging station $c_{k}$,

(21) $\quad d(\varsigma)=\sum_{(i, j) \in \varsigma} d_{i j}$

(22) $\quad e(\varsigma)=\sum_{(i, j) \in \zeta} e_{i j}$

(23) $\quad t(\varsigma)=t_{c}\left(c_{k}\right)+t_{w}\left(c_{k}\right)+\sum_{(i, j) \in \zeta} t_{d}(i, j)$

(24) fee $(\varsigma)=p_{c}\left(c_{k}\right) \cdot\left(E_{0}-E_{\mathrm{rem}} / \eta_{c_{k}}\right)$

(25) Calculate the driver preference $P=\left((\alpha \cdot d(\varsigma)+\beta \cdot t(\varsigma)+\gamma \cdot e(\varsigma)) \cdot(\mu \cdot \mathrm{fee}(\varsigma)) / \lambda_{c_{k}}\right)$.

(26) Select charging path and charging station of the smallest $P$, which is the final planned path recommended to the EV users.

Algorithm 3: The proposed EHBMA.

Initialization The particle swarm PSO is initialized with the population amount $m$. Position pos and velocity $V$ of each particle part $_{i}$ are initialized randomly in the feasible interval. part ${ }_{i} \cdot$ pBest and gBest are initialized as fit $\left(\operatorname{part}_{i} \cdot \operatorname{pos}\right) \operatorname{and}_{\max }\left(\right.$ fit $\left(\operatorname{part}_{i} \cdot \operatorname{pos}\right)$ ), respectively. The inertia factor $\omega$ and the acceleration constant $C_{1} C_{2}$ are initialized as constants.

(1) While $\max _{i}\left(\right.$ part $_{i}$.pos - part $_{i}$.previous_pos $)>\varepsilon$ or iteration_count $\leq$ MAX

(2) For each particle part p $_{i} \in \mathrm{PSO}$, update the position pos and the velocity $V$ as follows,

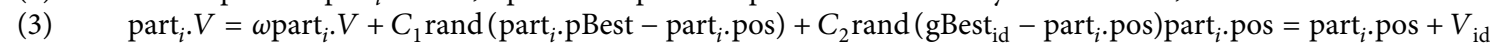

(4) If fit ( part $_{i} \cdot$ pos $) \geq$ fit $\left(\right.$ part $_{i} \cdot$ previous_pos $_{-}$, Then part $_{i} \cdot$ pBest $=$ fit $\left(\right.$ part $_{i} \cdot$ pos $)$

(5) If fit (part ${ }_{i} \cdot$ pos $) \geq$ fit (gBest), Then gBest $=$ fit $\left(\right.$ part $_{i} \cdot$ pos $)$

(6) $\quad$ part $_{i} \cdot$ previous_pos $=$ part $_{i}$.pos

(7) Output the optimal position gBest and optimized objective fit (gBest) 


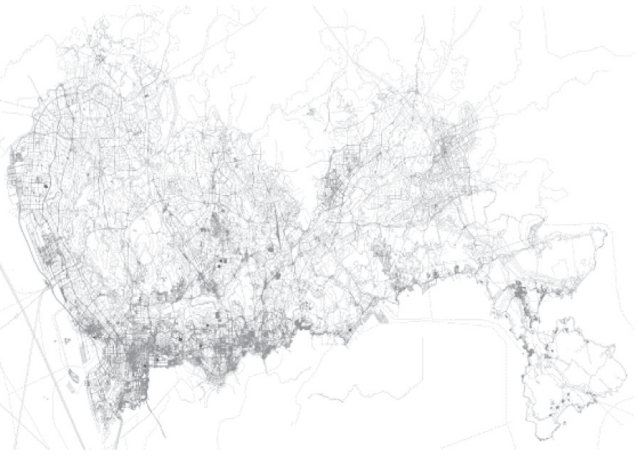

(a)

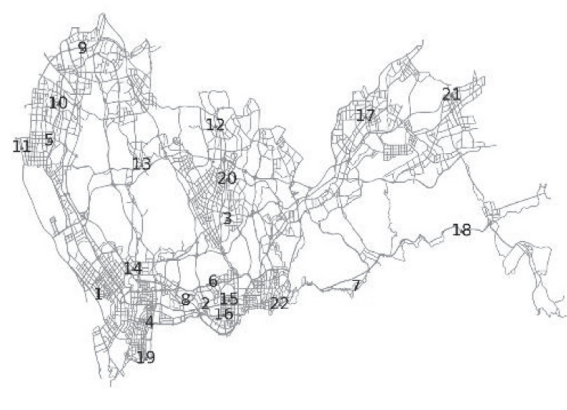

(b)

Figure 3: Road network of Shenzhen (2019): (a) original road network; (b) the selected road network with charging stations.

Table 3, and the used road network is shown in Figure 3(b) with charging stations marked on the figures. The driving roads are selected to construct a simplified road network, which includes 658,213 nodes, 132,182 edges, and 22 public charging stations.

The total amount of EVs in Shenzhen is about 272,687 (by $17^{\text {th }}$ Oct, 2019). Since most of the EVs are charged at private charging stations, we randomly select $1000 \mathrm{EVs}$ to simulate. The initial locations of EVs are randomly distributed at various nodes of the map, and the initial SOC is randomly set between $10 \%$ and $90 \%$. It is assumed that EVs will generate charging demand when SOC $\leq 20 \%$ and fully charged at the charging stations. The charging piles' number of the charging stations is randomly initialized in the interval $[10,50]$.

6.1. Comparison between MA, BMA, and Our Proposed EHBMA. MA, BMA, and EHBMA are performed to select nodes to the charging station. The execution time and the traversed node label number of the three algorithms are shown in Table 4. It is demonstrated that MA costs the longest time, and EHBMA costs the shortest time. The traversed nodes are marked with different colors in Figures 4-6, where the green points and the blue points represent the nodes which are searched forward and backward, respectively. It can be concluded that BMA traverses fewer nodes than MA, while the proposed EHBMA traverses least nodes than both of MA and BMA.

6.2. Single EV Charging Path Planning Simulation. The simulation parameters are set as follows. The charging price is fixed as $1.75 \mathrm{RMB} / \mathrm{kWh}$ for all charging stations. The driver preference factors are $\alpha=0.6, \beta=1800, \gamma=703$, and $\mu=0.02$, and the initial locations are randomly selected on the map with initial SOC $=20 \%$ and $E_{0}=70 \mathrm{kWh}$.

In Figure 7, the red, blue, and green paths are the charging paths to the three preselected charging stations, No. 8 , No. 13, and No. 12, respectively. The cost of the paths, as shown in Table 5, is used to calculate the driver's preference, through which the driving distance, energy consumption, time consumption, and charging fee are considered with a trade-off. Then, the path with the smallest $P$ value is selected,
TABLE 3: Speed limit of the roads.

\begin{tabular}{lc}
\hline Road type & Speed limit $(\mathrm{km} / \mathrm{h})$ \\
\hline Residential road & 20 \\
Tertiary road & 30 \\
Secondary road & 50 \\
Primary road & 90 \\
Trunk road & 100 \\
Motorway road & 120 \\
\hline
\end{tabular}

TABle 4: Performance comparison of MA, BMA, and EHBMA.

\begin{tabular}{lccc}
\hline & MA & BMA & EHBMA \\
\hline Computation time (s) & 46.57 & 6.28 & 0.31 \\
Number of the traversed nodes & 41297 & 13324 & 1552 \\
\hline
\end{tabular}

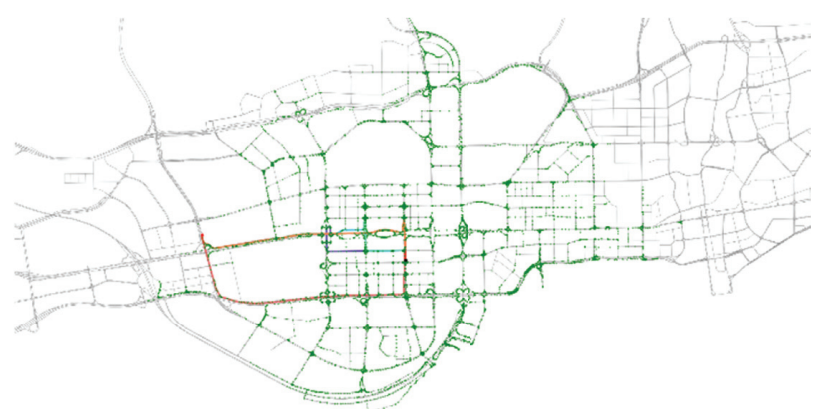

FIGURE 4: Charging path planning by MA.

so the green path to charging station No. 12 is the final planned charging path.

6.3. Large-Scale EVs Charging Path Planning Simulation. In this experiment, $1000 \mathrm{EVs}$ are randomly selected on the map. The simulation parameter settings are as follows. Charging price for all charging stations is set as a constant value, $1.75 \mathrm{RMB} / \mathrm{kWh}$. The charging threshold of EVs is set to SOC $=20 \%$. The battery capacity $E_{0}$ is randomly initialized between $50 \mathrm{kWh}$ and $90 \mathrm{kWh}$, and the driver preference factors are $\alpha=0.6, \beta=1800, \gamma=703$, and $\mu=0.02$.

The results of the large-scale EVs' path planning using EHBMA are shown in Figure 8. The total revenues of all 


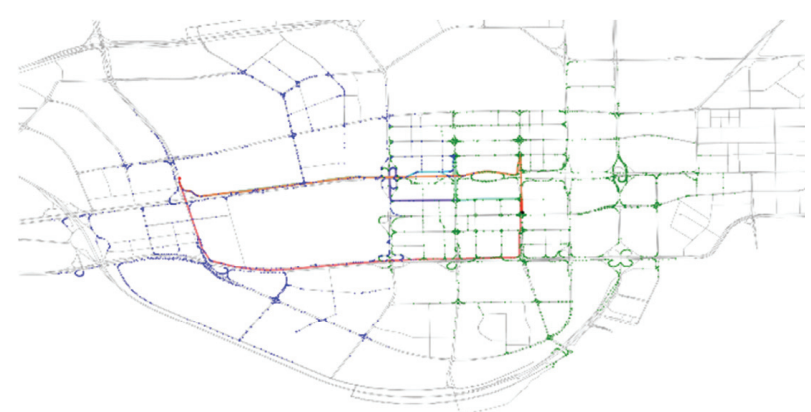

FIgure 5: Charging path planning by BMA.

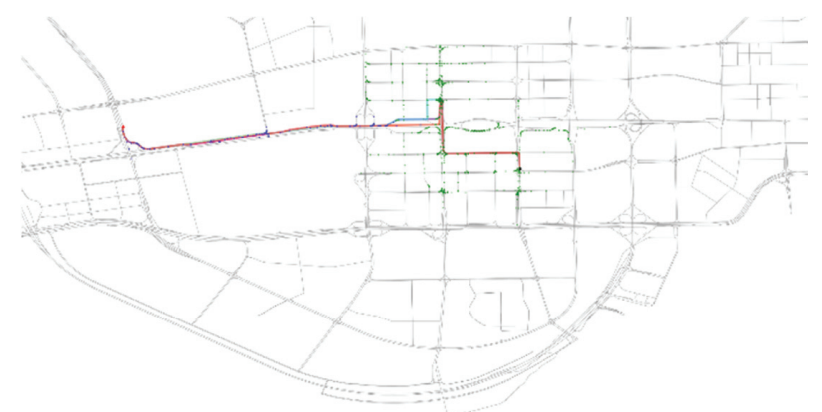

FIGURE 6: Charging path planning by EHBMA.

charging stations, revenue of each charging station, and EV charging amount of each charging station are listed in Tables 6-8, which demonstrate that the revenues and EV amount between different charging stations are not equal. Therefore, it is necessary to optimize the charging price to maximize the total revenue and balance the revenue of each charging station.

6.4. The Simulation of CSBAP. In this experiment, the population of the particle swarm in PSO is set as $m=20$, and the maximum number of iterations is set as $\mathrm{MAX}=100$.

Considering the pricing strategy for the single charging station, the No. 6 charging station is used as a case study. Table 9 lists the revenue of the charging station No. 6 when the prices of charging stations are fixed as the same price and the charging price is adjusted by CSBAP. The results show that, after the charging price of the charging station No. 6 is adjusted by CSBAP, more revenue can be obtained than the fixed charging price strategy, since the lower price would attract more EVs to charge. Therefore, the effectiveness of our proposed CSBAP for a single charging station is validated.

As the charging pricing strategy for all charging stations, the simulation conditions are set as follows. The prices of the charging stations are limited to the interval [1.5-2.0] RMB/ kWh. $m=20$, and MAX $=100$. The fitness function is defined as

$$
\text { fit }=\sum_{c_{i}}^{C} r\left(c_{i}\right)-10 \cdot \operatorname{std}\left(\widehat{r}\left(c_{i}\right)\right) \text {. }
$$

Figure 9 shows that the value of the fitness function gradually rises and eventually stabilizes with 100 iterations of the charging price. Figure 10 illustrates that, after the iterations of CSBAP, the total revenues of all charging stations gradually increase and eventually stabilize. The different color columns in Figure 10 represent the profits of different charging stations. Figure 11 demonstrates that, after the iterations of CSBAP, the revenue standard deviation of charging stations gradually decreases and eventually stabilizes, indicating that the revenues between charging stations are more balanced than those without optimization. Taking the charging price of one of the charging stations as an example, Figure 12 shows the iteration of the charging price of charging station No. 10, where the ordinates and the size of the circle represent the charging price and the revenue of the charging station, respectively, which illustrates that the charging price of charging station No. 10 decreases, while the revenues increase with the applied CSBAP.

Figures 13 and 14 are the planned charging paths of large-scale EVs before and after using CSBAP. Figures 15 and 16 are the charging prices without and with optimization of CSBAP, where the horizontal coordinates, the ordinates, and the size of the circles represent the 


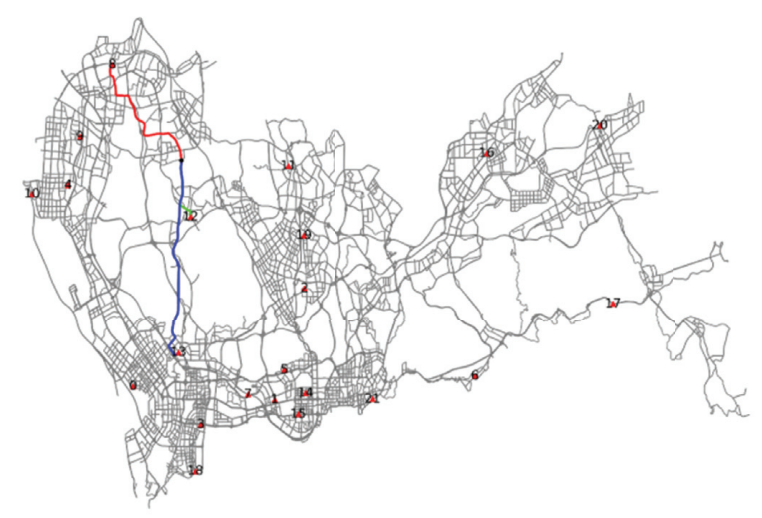

FIgURE 7: Charging path planning for single EV.

TABLE 5: Cost of the paths to three charging stations.

\begin{tabular}{lccccc}
\hline $\begin{array}{l}\text { Charging station } \\
\text { number }\end{array}$ & $\begin{array}{c}\text { Driving distance } \\
(\mathrm{km})\end{array}$ & $\begin{array}{c}\text { Time consumption } \\
(\mathrm{h})\end{array}$ & $\begin{array}{c}\text { Energy consumption } \\
(\mathrm{kWh})\end{array}$ & $\begin{array}{c}\text { Charging fee } \\
(\mathrm{RMB})\end{array}$ & $\begin{array}{c}\text { Driver preference } \\
P\end{array}$ \\
\hline 8 & 13416.536 & 2.146 & 2.173 & 87.803 & 51287.242 \\
12 & 5407.877 & 2.002 & 0.745 & 85.305 & 9370.217 \\
13 & 16814.498 & 2.231 & 2.661 & 88.658 & 75376.958 \\
\hline
\end{tabular}

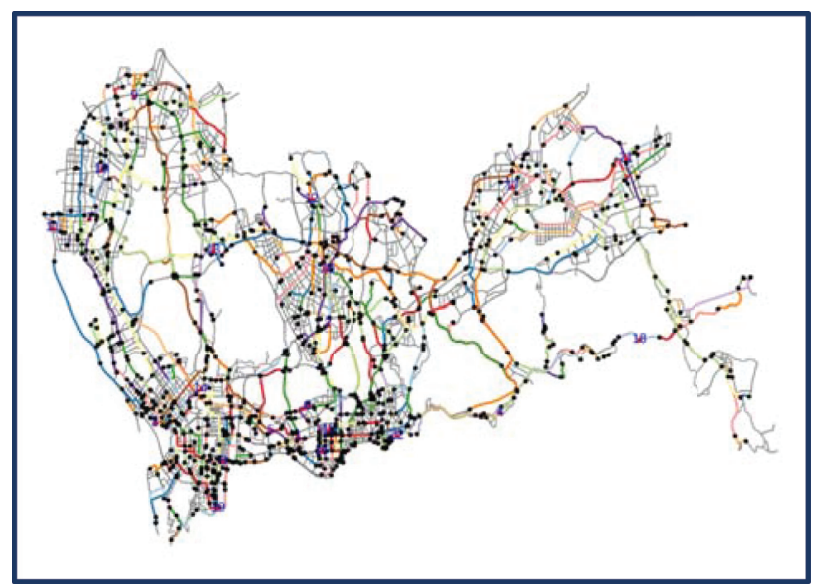

FIgURE 8: Path planning for large-scale EV users.

TABLE 6: Total revenues of charging stations based on large-scale EV path planning.

Total revenues of the charging station (RMB) Standard deviation of revenues for each charging station 85878.635 1312.150

TABLE 7: Revenue of each charging station.

\begin{tabular}{lccccccccccc}
\hline Number & 1 & 2 & 3 & 4 & 5 & 6 & 7 & 8 & 9 & 10 \\
\hline Revenue (RMB) & 5074.94 & 4248.56 & 4885.47 & 4960.57 & 4721.40 & 1023.80 & 3137.47 & 1628.30 & 3502.23 & 2125.78 & 1019.49 \\
Number & 12 & 13 & 14 & 15 & 16 & 17 & 18 & 19 & 20 & 21 & 22 \\
Revenue (RMB) & 3438.18 & 4818.88 & 4983.96 & 4698.36 & 4623.16 & 4821.92 & 3160.76 & 5060.51 & 4831.88 & 4310.15 & 4802.79 \\
\hline
\end{tabular}


TABLE 8: Charging EV amount of each charging station.

\begin{tabular}{lccccccccccc}
\hline Number & 1 & 2 & 3 & 4 & 5 & 6 & 7 & 8 & 9 & 10 \\
\hline EV amount & 59 & 50 & 57 & 58 & 55 & 12 & 36 & 19 & 41 & 25 \\
Number & 12 & 13 & 14 & 15 & 16 & 17 & 18 & 19 & 20 & 21 & 22 \\
EV amount & 40 & 56 & 58 & 55 & 54 & 56 & 36 & 59 & 56 & 50 & 56 \\
\hline
\end{tabular}

TABLE 9: Revenue of the charging station No. 6.

\begin{tabular}{lccc}
\hline Pricing strategy & Charging price $(\mathrm{RMB} / \mathrm{kWh})$ & Revenue & EV amount \\
\hline Fixed charging price & 1.79 & 1863.05 & 21 \\
CSBAP & 1.61 & 2049.07 & 23 \\
\hline
\end{tabular}

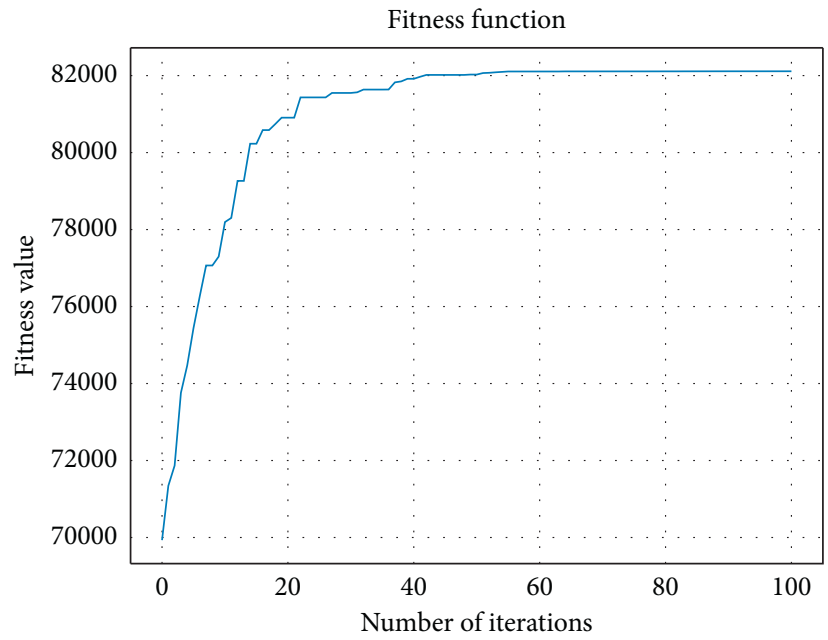

Figure 9: Fitness value iteration.

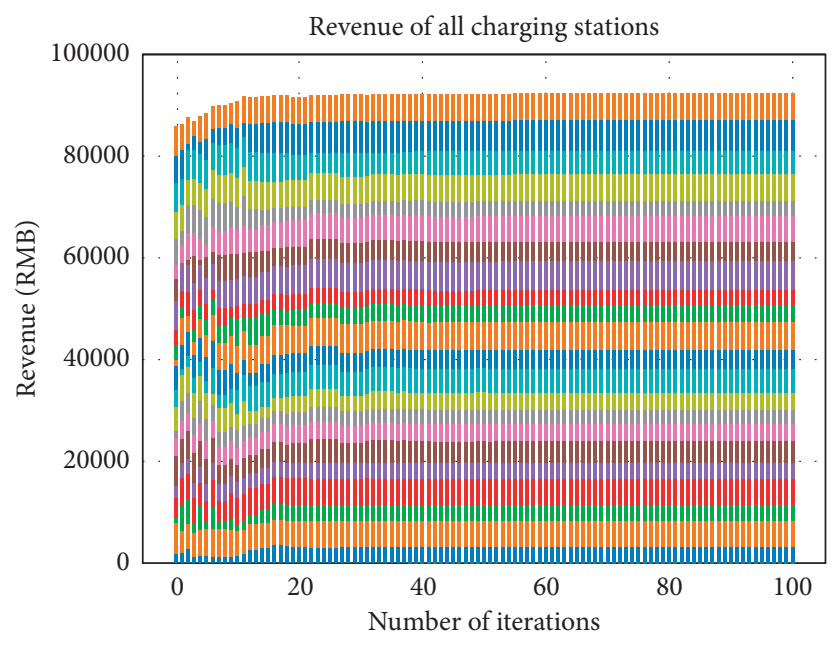

FIGURE 10: Revenue iteration of charging stations.

number of the charging station, the charging price, and the revenue, respectively. It can be observed that the circles in Figure 16 are more similar in size than circles in
Figure 15, which represents that revenues of the charging stations are more balanced with CSBAP without decreasing the total revenues of the charging stations. 


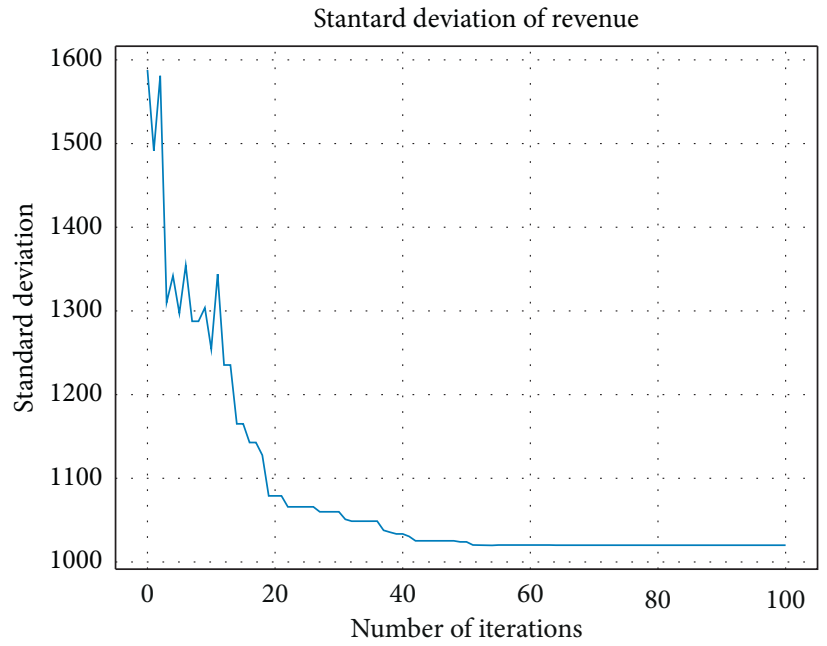

FIgURE 11: Standard deviation of revenues.

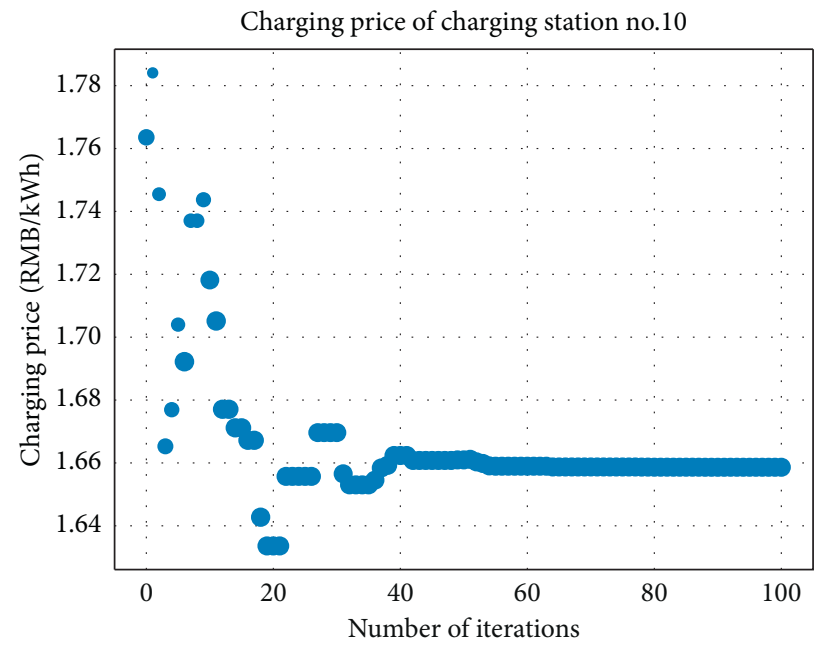

Figure 12: Charging price of charging station No. 10.

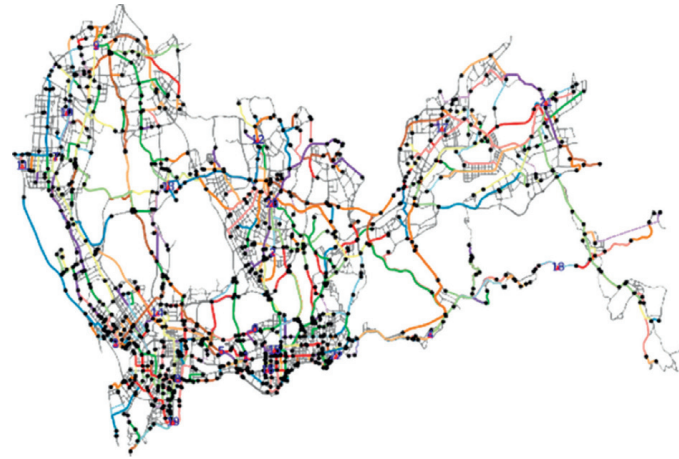

Figure 13: Planned path before optimization. 


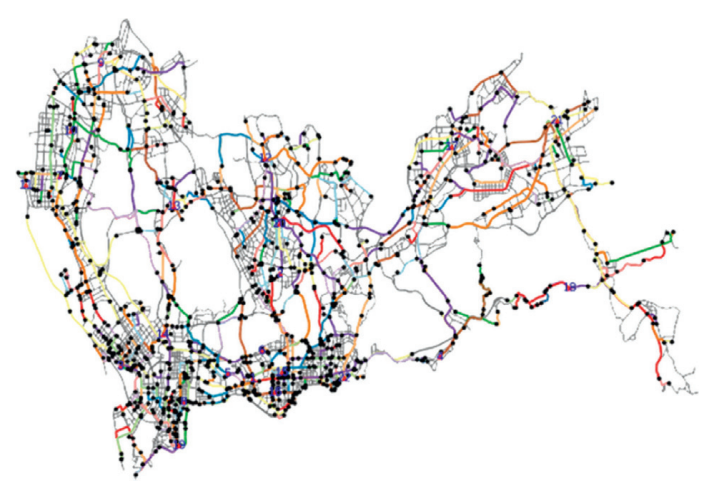

Figure 14: Planned path after optimization.

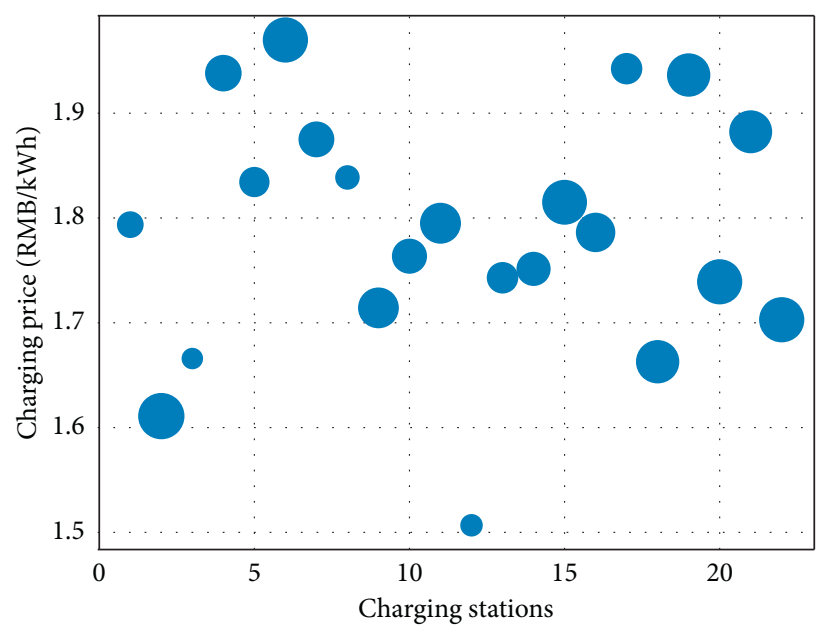

FIGURE 15: Charging price without optimization.

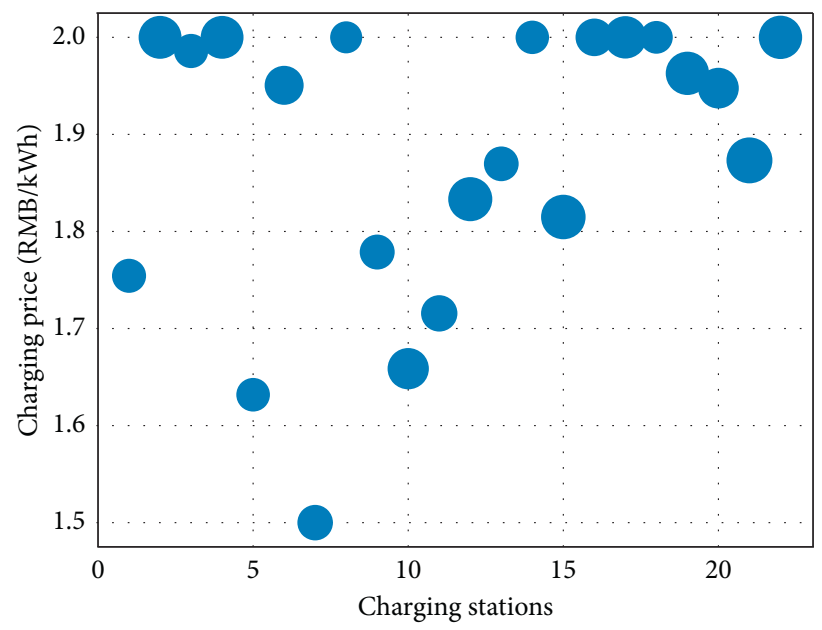

FIGURE 16: Charging price with optimization. 


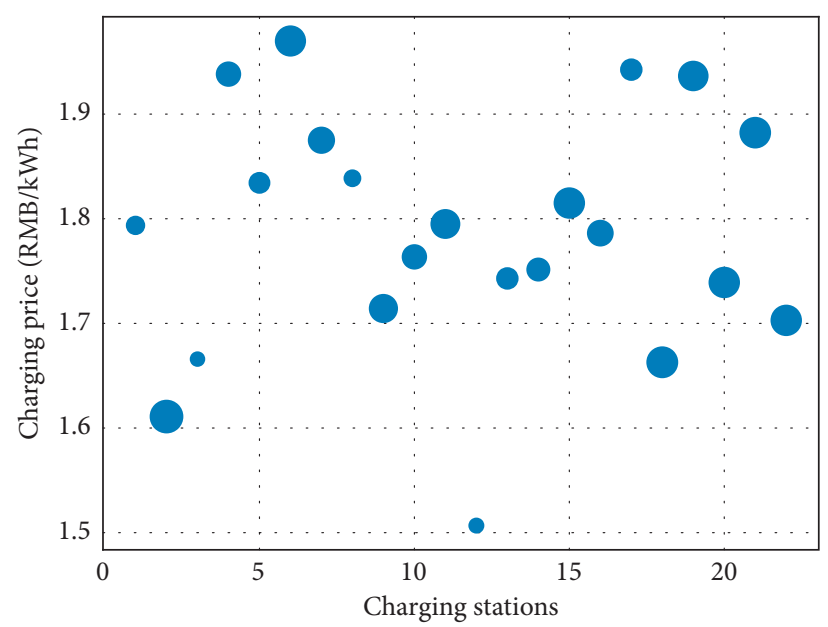

FIgURE 17: Charging price without optimization.

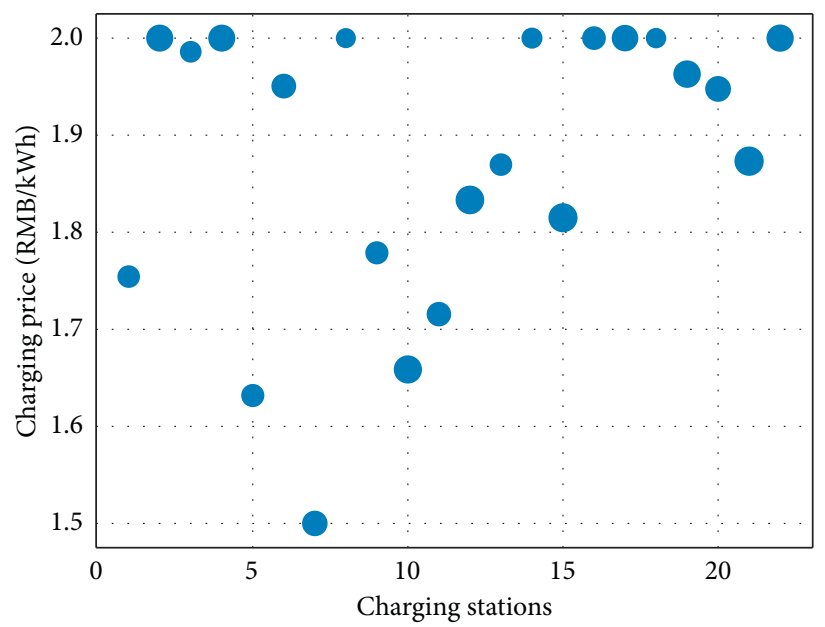

FIGURE 18: Charging price with optimization.

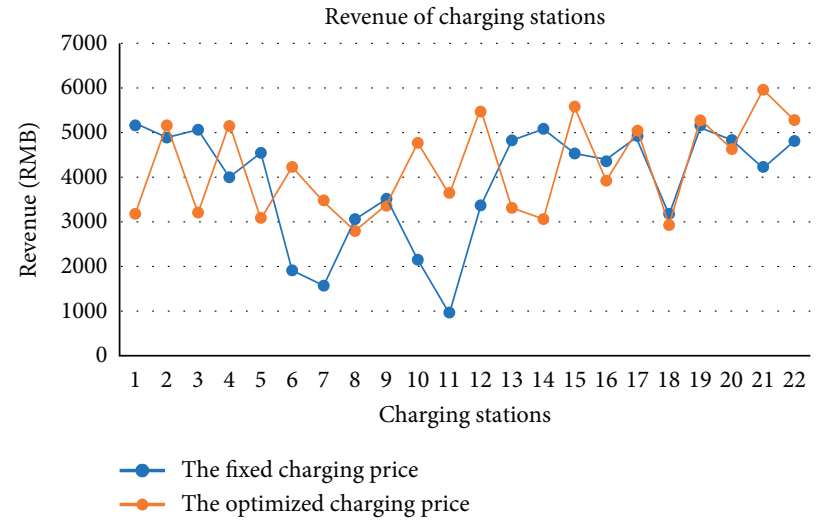

FIgURE 19: Comparison of revenue. 


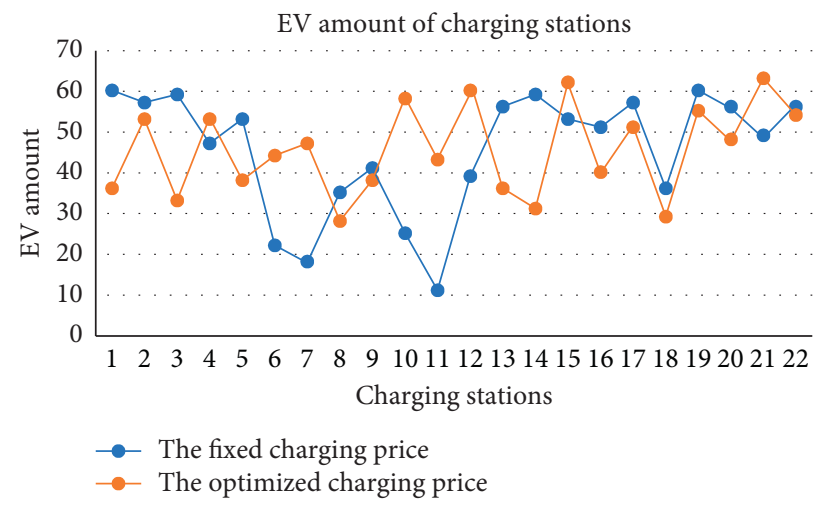

Figure 20: Comparison of EV amount.

TABLE 10: Comparison between fixed pricing strategy and CSBAP.

\begin{tabular}{lcc}
\hline & Fixed pricing strategy & CSBAP \\
\hline Revenue (RMB) & 85869.24 & 92312.38 \\
Standard deviation of revenue between charging stations & 1258.79 & 1020.03 \\
Standard deviation of EV amount between charging stations & 14.64 & 10.73 \\
\hline
\end{tabular}

Figures 17 and 18 are the comparison of the amount of charging EVs at the charging stations without and with optimization of CSBAP, where the horizontal coordinates, the ordinates, and the size of the circles represent the number of the charging station, the charging price, and the charged EV amount in the charging stations, respectively. Figures 19 and 20 are the comparison results of the revenue of charging stations and EV amount between using fixed charging price (i.e., charging price is fixed as $1.75 \mathrm{RMB} / \mathrm{kWh}$ ) and CSBAP. It is demonstrated from Figures 19 and 20 and Table 10 that, by using CSBAP, charging stations gain more revenues than using the fixed charging price, while the revenues and EV amount of charging stations are more balanced. According to the simulation results, the effectiveness of the proposed CSBAP for charging stations is verified.

\section{Conclusion}

In this paper, an integrated framework of combining the multiobjective charging path planning method is discussed for EVs and charging station pricing strategy for charging stations. A novel charging path planning algorithm EHBMA is proposed based on bidirectional Martins' algorithm, considering driving distance, energy consumption, total time consumption, and charging fee. It is verified by simulation experiments that the proposed algorithm can provide more charging paths for EV users and assist EV users to select charging stations and charging path with the driver's preferences. Furthermore, a novel charging pricing strategy CSBAP is proposed. It is also verified by simulation experiments that the revenues of charging stations can be effectively improved, while the revenues of the charging stations are balanced by using the proposed pricing strategy.
In the future, we will study the travel patterns of EVs and how to create user portraits to improve the appropriateness of the recommended charging paths. The potential of combining the market transaction mechanism to determine the charging price of charging stations will be investigated. Moreover, more complex road conditions and driving conditions of EVs should be considered to obtain a more accurate charging path planning model.

\section{Data Availability}

The data used to support the study are available from Openstreetmap (https://www.openstreetmap.org/\#map=11/ 22.5497/114.0436).

\section{Conflicts of Interest}

The authors declare that there are no conflicts of interest regarding the publication of this paper.

\section{Acknowledgments}

This work was partially supported under the Natural Science Foundation of Guangdong Province Ref. 2018A030313755 and Shenzhen Fundamental Research Program Ref. JCYJ20200109114839874.

\section{References}

[1] G. Wei, F. Lei, R. Lin et al., "Algorithms for probabilistic uncertain linguistic multiple attribute group decision making based on the GRA and CRITIC method: application to location planning of electric vehicle charging stations," Economic Research-Ekonomska Istraživanja, vol. 33, no. 1, pp. 828-846, 2020. 
[2] J. Bi, Y. Wang, Q. Sai, and C. Ding, "Estimating remaining driving range of battery electric vehicles based on real-world data: a case study of Beijing, China," Energy, vol. 169, pp. 833-843, 2019.

[3] X.-G. Yang and C.-Y. Wang, "Understanding the trilemma of fast charging, energy density and cycle life of lithium-ion batteries," Journal of Power Sources, vol. 402, pp. 489-498, 2018.

[4] F. Fu and H. Dong, "Targeted optimal-path problem for electric vehicles with connected charging stations," PLoS One, vol. 14 , no. 8, pp. 1-23, 2019.

[5] M. Alizadeh, H. T. Wai, M. Chowdhury, A. Goldsmith, A. Scaglione, and T. Javidi, "Optimal pricing to manage electric vehicles in coupled power and transportation networks," IEEE Transactions on Control of Network Systems, vol. 4, no. 4, 2017.

[6] M. Duell, L. M. Gardner, and S. T. Waller, "Policy implications of incorporating distance constrained electric vehicles into the traffic network design problem," Transportation Letters, vol. 10, no. 3, pp. 144-158, 2018.

[7] M. S. Rahaman, Y. Mei, M. Hamilton, and F. D. Salim, "CAPRA: a contour-based accessible path routing algorithm," Information Sciences, vol. 385-386, pp. 157-173, 2017.

[8] P. Barbecho Bautista, L. Lemus Cárdenas, L. Urquiza Aguiar, and M. Aguilar Igartua, "A traffic-aware electric vehicle charging management system for smart cities," Vehicular Communications, vol. 20, Article ID 100188, 2019.

[9] J. Liu, H. Guo, J. Xiong, N. Kato, J. Zhang, and Y. Zhang, "Smart and resilient EV charging in SDN-enhanced vehicular edge computing networks," IEEE Journal on Selected Areas in Communications, vol. 38, no. 1, 2020.

[10] H. Zhang, Y. Zhou, Q. Zheng et al., "A novel large group decision making method via normalized alternative prediction selection," IEEE Transactions on Fuzzy Systems, vol. 6706, p. 1, 2020.

[11] Z. Chen, M. Lu, Y. Zhou, and C. Chen, "Information synergy entropy based multi-feature information fusion for the operating condition identification in aluminium electrolysis," Information Sciences, vol. 548, pp. 275-294, 2021.

[12] Y. C. Zheng, J. Wang, D. Guo et al., "Study of multi-objective path planning method for vehicles," Environmental Science and Pollution Research, vol. 27, no. 3, pp. 3257-3270, 2020.

[13] X. Zhang, P. Li, J. Hu et al., "Yen's algorithm-based charging facility planning considering congestion in coupled transportation and power systems," IEEE Transactions on Transportation Electrification, vol. 5, no. 4, pp. 1134-1144, 2019.

[14] S. Zhang, M. Chen, and W. Zhang, "A novel location-routing problem in electric vehicle transportation with stochastic demands," Journal of Cleaner Production, vol. 221, pp. 567581, 2019.

[15] W. Mei Gai, Y. Feng Deng, Z. An Jiang, J. Li, and Y. Du, "Multi-objective evacuation routing optimization for toxic cloud releases," Reliability Engineering and System Safety, vol. 159, pp. 58-68, 2017.

[16] E. Q. V. Martins, "On a multicriteria shortest path problem," European Journal of Operational Research, vol. 16, no. 2, pp. 236-245, 1984.

[17] S. Demeyer, J. Goedgebeur, P. Audenaert, M. Pickavet, and P. Demeester, "Speeding up Martins' algorithm for multiple objective shortest path problems," $4 O R$, vol. 11 , no. 4 , pp. 323-348, 2013.

[18] M. Tegnér, R. R. Ernstsen, A. Skajaa, and R. Poulsen, "Riskminimisation in electricity markets: fixed price, unknown consumption," Energy Economics, vol. 68, pp. 423-439, 2017.
[19] H. Yang, Z. Gong, Y. Ma, L. Wang, and B. Dong, "Optimal two-stage dispatch method of household PV-BESS integrated generation system under time-of-use electricity price," International Journal of Electrical Power \& Energy Systems, vol. 123, Article ID 106244, 2020.

[20] S. Nojavan, K. Zare, and B. Mohammadi-Ivatloo, "Optimal stochastic energy management of retailer based on selling price determination under smart grid environment in the presence of demand response program," Applied Energy, vol. 187, pp. 449-464, 2017.

[21] Z. Wang and R. Paranjape, "Optimal residential demand response for multiple heterogeneous homes with real-time price prediction in a multiagent framework," IEEE Transactions on Smart Grid, vol. 8, no. 3, pp. 1173-1184, 2017.

[22] R. Khatami, M. Heidarifar, M. Parvania, and P. Khargonekar, "Scheduling and pricing of load flexibility in power systems," IEEE Journal of Selected Topics in Signal Processing, vol. 12, no. 4, pp. 645-656, 2018.

[23] Y. Liu, R. Deng, and H. Liang, "A stochastic game approach for PEV charging station operation in smart grid," IEEE Transactions on Industrial Informatics, vol. 14, no. 3, pp. 969-979, 2018.

[24] T. Z. Zhang and T. D. Chen, "Smart charging management for shared autonomous electric vehicle fleets: a Puget Sound case study," Transportation Research Part D Transport and Environment, vol. 78, Article ID 102184, 2020.

[25] F. Abbas, D. Feng, S. Habib, M. Numan, and U. Rahman, "A heuristically optimized comprehensive charging scheme for large-scale EV integration," International Transactions on Electrical Energy Systems, vol. 30, no. 2, pp. 1-21, 2020.

[26] X. Xu, D. Niu, Y. Li, and L. Sun, "Optimal pricing strategy of electric vehicle charging station for promoting green behavior based on time and space dimensions," Journal of Advanced Transportation, vol. 2020, Article ID 8890233, 2020.

[27] Z. J. Lee, J. Z. F. Pang, and S. H. Low, "Pricing EV charging service with demand charge," Electric Power Systems Research, vol. 189, Article ID 106694, 2020.

[28] C. Latinopoulos, A. Sivakumar, and J. W. Polak, "Response of electric vehicle drivers to dynamic pricing of parking and charging services: risky choice in early reservations," Transportation Research Part C: Emerging Technologies, vol. 80, pp. 175-189, 2017.

[29] Z. Lu, J. Qi, J. Zhang, L. He, and H. Zhao, "Modelling dynamic demand response for plug-in hybrid electric vehicles based on real-time charging pricing," IET Generation, Transmission \& Distribution, vol. 11, no. 1, pp. 228-235, 2017.

[30] L. Shen, H. Shao, T. Wu, W. H. K. Lam, and E. C. Zhu, "An energy-efficient reliable path finding algorithm for stochastic road networks with electric vehicles," Transportation Research Part C Emerging Technologies, vol. 102, pp. 450-473, 2019.

[31] D. Said, S. Cherkaoui, and L. Khoukhi, "Queuing model for EVs charging at public supply stations," in Proceedings of the 2013 9th International Wireless Communications and Mobile Computing Conference (IWCMC), pp. 65-70, Sardinia, Italy, July 2013. 